\title{
Criminologie
}

\section{La détention provisoire au Québec : éléments de connaissance et propositions de réflexions}

\section{Marie-Marthe Cousineau}

Volume 28, numéro 2, 1995

La détention provisoire

URI : https://id.erudit.org/iderudit/017370ar

DOI : https://doi.org/10.7202/017370ar

Aller au sommaire du numéro

\section{Éditeur(s)}

Les Presses de l'Université de Montréal

ISSN

0316-0041 (imprimé)

1492-1367 (numérique)

Découvrir la revue

Citer cet article

Cousineau, M.-M. (1995). La détention provisoire au Québec : éléments de connaissance et propositions de réflexions. Criminologie, 28(2), 5-26. https://doi.org/10.7202/017370ar
Résumé de l'article

For a long time, it has been argued that pre-trial detention should be used with moderation, only in those occasions where no other means seems sufficient to insure the presence of the suspect at his trial or to warrant the security of the public during the duration of the criminal proceedings. Looking at the official statistics reported by the author, one could easily conclude that, in Canada, the pre-trial detention is still largely used. By following a cohort of 1792 suspects from the moment they were apprehended by the MUC police agents in 1989, till the end of Court procedures, the author identifies the characteristics of the suspects who were maintained in pre-trial detention in comparison to the ones that were not submitted to this measure. Further, the author addresses the controversial question of the consequences of pre-trial detention on penal issues. In conclusion the author raises the question if it could be possible to develop other ways of dealing with what she defines as the perceived risk that a suspect tries escape trial or continues to cause harm to the society during the trial. 


\begin{abstract}
For a long time, it has been argued that pre-trial detention should be used with moderation, only in those occasions where no other means seems sufficient to insure the presence of the suspect at his trial or to warrant the security of the public during the duration of the criminal proceedings. Looking at the official statistics reported by the author, one could easily conclude that, in Canada, the pre-trial detention is still largely used. By following a cohort of 1792 suspects from the moment they were apprehended by the MUC police agents in 1989, till the end of Court procedures, the author identifies the characteristics of the suspects who were maintained in pre-trial detention in comparison to the ones that were not submitted to this measure. Further, the author addresses the controversial question of the consequences of pre-trial detention on penal issues. In conclusion the author raises the question if it could be possible to develop other ways of dealing with what she defines as the perceived risk that a suspect tries escape trial or continues to cause harm to the society during the trial.
\end{abstract}

Les admissions de personnes dans les établissements de détention du Québec $^{2}$ concernent essentiellement deux types de clientèles : 1) les gens admis pour avoir omis de payer une amende leur ayant été initialement imposée à titre de sentence sanctionnant une infraction, le plus souvent aux lois de la circulation routière, et 2 ) les personnes incarcérées pour une partie, voire parfois pour la durée des procédures, des personnes, donc,

1. L'auteure détient une mâitrise en criminologie de l'Université de Montréal et un doctorat en sociologie de l'Université du Québec à Montréal. Elle est actuellement professeure adjointe à l'École de criminologie, Université de Montréal, C.P. 6128, Succ. Centre-ville, Montréal, Qc, H3C 3J7. Elle assure la codirection du Groupe de recherche et d'analyse sur les politiques et les pratiques pénales (GRAPPP).

2. Au Canada, on trouve deux types d'institutions carcérales : les pénitenciers qui admettent les accusés condamnés à de longues peines (deux ans et plus) et les prisons qui admettent deux types de clientèle, soit les personnes condamnées à de courtes peines (deux ans moins un jour ou moins) et les personnes détenues pour une partie ou toute la durée des procédures pénales qui les concernent (les détenus provisóires dont il est essentiellement question dans cet article). 
soumises à des mesures de détention avant même que leur culpabilité n'ait été reconnue ${ }^{3}$.

Il s'agit là de deux problématiques - l'incarcération pour nonpaiement d'amende et la détention provisoire - qui mettent en évidence le caractère discriminatoire de la prison à l'égard des plus démunis ${ }^{4}$. Dans le premier cas, des individus sont incarcérés parce qu'ils sont, pour une majorité d'entre eux, incapables de payer une amende pourtant ordinairement peu élevée. Dans le second cas, qui nous intéresse ici plus particulièrement, des individus se retrouvent détenus avant même d'être reconnus coupables, non pas tant, nous le verrons, parce que la gravité de l'infraction dont ils sont soupçonnés présente un danger imminent pour la société, mais simplement parce qu'il se voient incapables de fournir des garanties de représentation $^{5}$ ou une caution ${ }^{6}$ suffisante pour que l'on juge qu'ils ne risquent pas de se soustraire à la justice en ne se présentant pas à leur procès.

Or, si le premier groupe, celui des personnes incarcérées pour nonpaiement d'amende, a fait l'objet de nombreux travaux au Québec au cours des dernières années ${ }^{7}$, le second a été, au cours de la même période, dans une large mesure ignoré des chercheurs ${ }^{8}$. Une étude que j'ai menée, qui consistait à suivre le cheminement d'une cohorte justiciable à travers toutes les étapes du processus judiciaire, $m$ 'a conduite à m'intéresser plus particulièrement à cette question de l'usage de la détention avant et durant le procès car il est rapidement apparu qu'il s'agissait là d'une plaque

3. Il est bien entendu qu'il s'agit ici des admissions en détention (le flux). Une analyse réalisée sur la base des populations carcérales présentes dans l'un ou l'autre des établissements de détention du Québec (les stocks, à défaut d'une meilleure appellation) donnerait un tout autre portrait. L'un comme l'autre sont le reflet de la réalité, une réalité différente.

4. Voir à ce sujet les travaux du GRAPPP, amorcés par Cousineau, Laberge et Théorêt (1986) et poursuivis par Néron (1988), sur la question plus spécifique de l'incarcération pour non-paiement d'amendes et par Garceau (1988), sur celle de la détention provisoire.

5. Sous cette appellation, couramment employée par les professionnels de la justice pénale en Europe notamment et qui nous semble bien traduire ce qu'il en est, nous trouvons certains indicateurs de la situation sociale et matérielle des personnes mises en cause à partir desquelles ces agents évaluent le risque que ces dernières ne s'enfuient avant leur procès : il s'agit de la possession d'un domicile, d'un emploi, d'une famille... (À ce sujet, voir Levy (1987) entre autres).

6. Une caution n'est pas nécessairement monétaire, bien qu'elle puisse l'être. II peut aussi s'agir de l'engagement d'un tiers à s'assurer que l'inculpé se conformera aux conditions de sa remise en liberté et se présentera au tribunal torsque requis.

7. Voir, entre autres, les travaux de Hattem (1980), Néron (1988), Landreville (1994), et Casavant (à paraître).

8. À deux exceptions près : l'étude de Garceau (1988) qui porte spécifiquement sur la détention provisoire et celle de Cardinal (1989) qui s'intéresse au recours à la mise sous garde par les policiers. 
tournante du processus pénal. En effet, en suivant 1792 suspects à travers les méandres de leur parcours pénal, j'ai pu voir clairement que des trajectoires différentes se dessinaient suivant le statut de l'inculpé, c'est-à-dire suivant qu'il se présente à son procès, libre ou détenu.

En premier lieu, dans le cadre de cet article, après avoir présenté l'état de la recherche ainsi que les positions officielles soutenues au fil des ans sur la question du recours à la détention avant et durant le procès, je brosserai le portrait statistique de l'usage de cette mesure, au Québec, au cours des quinze années s'échelonnant entre 1977 et 1991 (dernière année pour laquelle on dispose d'informations). Cette première description me permettra d'en établir clairement l'importance, ne serait-ce qu'en termes d'occurrences.

En second lieu, je montrerai que la décision de mettre en détention provisoire est loin de constituer une mesure isolée. Elle est partie d'un processus qui, d'une part, est influencé par l'amont (ce qui s'est passé avant) et, d'autre part, influence en aval le traitement de la plainte. Bref, il s'agit d'une mesure-charnière à la fois déterminée et déterminante. Je m'attarderai donc, dans un premier temps, sur cette première partie de la proposition qui en fait une mesure déterminée. Je chercherai ainsi à mettre en lumière les facteurs associés à l'imposition d'une mesure de détention provisoire ou, à l'inverse, à la décision de remise en liberté du suspect pour une partie ou pour la durée des procédures. Je me pencherai, dans un deuxième temps, sur cette partie de la proposition qui fait de la détention provisoire une mesure ayant une influence déterminante sur le cours du processus pénal, et plus particulièrement sur la prise de décision judiciaire sur la cause et sur la sentence.

\section{UN ÉTAT DE LA RECHERCHE}

Mon propos s'inspire ici largement d'une recension d'écrits réalisée par Landreville et Laberge (1993) qui me paraît résumer fort justement l'état de la question. Constatant la rareté des écrits portant sur le sujet, les auteurs identifient néanmoins trois thèmes de recherche circonscrivant bien les discussions entourant la problématique de la détention avant et durant procès (ou détention prédécisionnelle ou détention provisoire). Il s'agit 1) des études portant sur les facteurs de décision; 2) des études évaluatives sur la non-comparution à son procès et sur la récidive; et 3 ) des études portant sur l'impact de la mesure.

Les études portant sur les facteurs présidant aux décisions des agents pénaux en matière d'incarcération avant et durant procès permettent d'identifier le type d'informations retenues et la manière suivant laquelle celles-ci affectent la décision de mise sous garde. Landreville et Laberge (1992: 45) 
constatent que les résultats statistiques des différentes études réalisées sur ce thème convergent sur certains points : on note, entre autres, que non seulement la nature du délit et l'histoire criminelle antérieure du suspect, mais aussi des éléments de stabilité sociale s'associant aux caractéristiques personnelles (sexe, âge, occupation et statut civil du suspect) influencent la décision des agents pénaux en matière de détention prédécisionnelle. Toutefois, tous ces facteurs ne suffisent pas à expliquer la variation observée dans les décisions rendues par le tribunal. D'autres considérations, difficilement identifiables, interviendraient selon les magistrats, les régions, voire les époques.

Les études évaluatives sur la non-comparution à son procès et sur la récidive insistent, pour leur part, sur la difficulté de prédire le comportement de l'inculpé. Or, c'est exactement sur cette question que sont appelés à se prononcer les agents pénaux au moment de décider du statut qui sera accordé au suspect pour la durée des procédures. «La plupart de ces prédictions, remarquent Landreville et Laberge (1993: 13), se font d'une façon implicite et intuitive et reposent sur l'expérience, les perceptions, les connaissances plus ou moins étendues et les préjugés des décideurs". Mais d'autres bases de prédiction, plus systématiques, sont éventuellement mises à contribution (bases cliniques, statistiques ou actuarielles). «De façon générale, énoncent les auteurs (idem: 16), on peut affirmer que les prédictions intuitives des décideurs de l'administration de la justice sont moins bonnes que celles des cliniciens, et que les leurs sont inférieures aux prédictions statistiques ou actuarielles". Mais aucune ne représente la panacée : le potentiel prédictif des critères retenus ne s'est jamais révélé très convaincant quelle que soit l'approche adoptée, qu'on cherche à prédire la non-comparution, la récidive ou la manifestation d'un comportement violent (Morden, 1980 ; Nuffield, 1982 ; Gottfredson et Gottfredson, 1986).

Dans tous les cas, la prédiction est susceptible de mener à deux types d'erreurs : on peut prédire qu'une personne récidivera ou sera violente alors qu'elle ne va pas l'être (faux positifs) ou bien on peut prédire qu'une personne ne récidivera pas ou ne sera pas violente alors qu'elle va l'être (faux négatifs). Landreville et Laberge (1993: 19) soulignent les conséquences de ces deux types d'erreurs : «[...] dans le premier cas, il se peut que l'on ait mis une personne en détention provisoire ou qu'on ait refusé de la libérer, pour prévenir un danger inexistant, ce qui pose un problème concernant les droits individuels. Dans le second cas, il peut y avoir une remise en liberté provisoire ou une libération conditionnelle qui sera suivie d'une récidive ou d'un comportement de violence".

Dans les faits, devant la difficulté à obtenir une prédiction vraiment efficace, il semblerait que les agents pénaux préfèrent ne pas prendre de 
risques et privilégient la détention aussitôt que la situation du contrevenant paraît un tant soit peu - véritablement ou potentiellement — problématique (Morden, 1980). Dans ces conditions, il n'est pas surprenant de constater l'ampleur que prend le recours à la détention prédécisionnelle, laquelle n'est pas sans conséquences, comme nous le verrons.

Enfin deux thèses, issues des études concernant l'impact de la détention provisoire sur le résultat des procédures pénales, s'affrontent. D'un côté, la thèse de la causalité (causal model) rend le statut de l'inculpé directement responsable de l'issue plus ou moins favorable de la cause (Friedland, 1965; P. Robert, 1986). De l'autre côté s'inscrit la thèse prônant l'existence d'une relation en quelque sorte artificielle (spurious model), entre tout un ensemble de variables intermédiaires liées d'abord au statut - en fait déterminant celui-ci - et seulement ensuite à l'issue des procédures. Les défenseurs de cette seconde thèse avancent que bien qu'ils soient à première vue Jiés, le statut de l'inculpé et le traitement de l'affaire constitueraient en réalité deux dimensions parallèlement influencées par toute une série de déterminants communs, parmi lesquels on retrouve, entre autres, la gravité de l'infraction, le nombre de chefs d'accusation, les antécédents judiciaires, l'existence de causes pendantes et le fait d'être déjà sous le coup d'une condamnation (ordonnance de probation, libération conditionnelle...). Cela suppose que les mêmes variables dans un premier temps expliquent la décision des agents pénaux de garder le prévenu en détention provisoire pour une partie ou toute la durée des procédures et, dans un second temps, justifient la sévérité accrue manifestée à l'endroit du contrevenant.

Mais, quelle que soit leur position, qu'ils jugent que le lien entre le statut de l'inculpé et l'issue des procédures soit direct ou artificiel, les auteurs s'entendent pour reconnaître le caractère pénalisant de la mesure elle-même.

\section{DES POSITIONS OFFICIELLES}

En 1969, le Comité canadien sur la réforme pénale et correctionnelle, appelé à prendre position sur le bien-fondé du recours à l'incarcération avant et durant le procès, déclarait :

La détention pendant cette période d'attente peut causer un tort injustifié à l'inculpé qui sera par la suite acquitté, et inutile à celui qui sera par la suite reconnu coupable [...] L'incarcération antérieure au procès peut faire perdre son emploi à l'accusé et, partant, l'empêcher de remplir ses obligations familiales et autres... Par voie de conséquence, cet état de choses peut affaiblir ses relations familiales et sociales [...] Ainsi, la période d'incarcération peut marquer à jamais l'accusé, même s'il est reconnu innocent par 
la suite [...] En outre, bon nombre des établissements servant à détenir ceux qui attendent leur procès sont vieux et mal aménagés. Les conditions de vie et d'hygiène y sont primitives. Il est difficile d'y pratiquer la ségrégation, et les mesures de sécurité destinées aux détenus les plus difficiles doivent s'appliquer à tous [...] Enfin les programmes y sont presque inexistants (Comité canadien sur la réforme pénale et correctionnelle, 1969: 108-109).

Pour toutes ces raisons, le Comité recommandait que l'on s'efforce de réduire, dans toute la mesure du possible, l'arrestation et la détention sans nécessité des personnes suspectes, et que l'on recoure à l'incarcération prédécisionnelle uniquement lorsqu'une telle mesure est absolument indispensable pour la protection de la société.

En 1988, la Commission de réforme du droit du Canada insistait pour sa part sur le fait que «le principe de l'équité exige que la détention ne soit utilisée qu'en dernier recours » (1988:30). En outre, précisent les membres de la Commission, "la procédure suivie pour décider de la mise en liberté ou du placement en détention devrait être conforme aux garanties qu'offre la Charte canadienne des droits et libertés notamment le droit à l'égalité et le droit à la liberté, dans toute la mesure du possible".

Or, malgré les recommandations émanant de ces différents comités d'étude, qui reposent sur des constats qui sont loin d'être nouveaux, il apparaît clairement que l'on persiste, encore aujourd'hui, à recourir dans de très nombreuses situations à ce type d'incarcération.

\section{UN ÉTAT DE LA SITUATION AU QUÉBEC}

L'ampleur du recours à la détention provisoire, au Québec comme ailleurs, a depuis longtemps été reconnue. En fait, la proportion d'admissions de détenus provisoires dans les prisons du Québec est non seulement importante, mais elle est en continuelle progression. Cela se vérifie depuis au moins le début des années 1980, de sorte qu'en 1986, la proportion des admissions de prévenus au sein des institutions carcérales de juridiction québécoise surpasse celles des admissions de condamnés. Au tournant des années 1990, les prévenus représentent plus de $60 \%$ des entrées dans les prisons québécoises. Il ne s'agit donc pas, comme le sous-entend sa définition, d'une mesure exceptionnelle (figure 1). 
Figure 1

Distribution des admissions en détention provisoire et en détention punitive dans les établissements de détention du Québec, 1977-1991

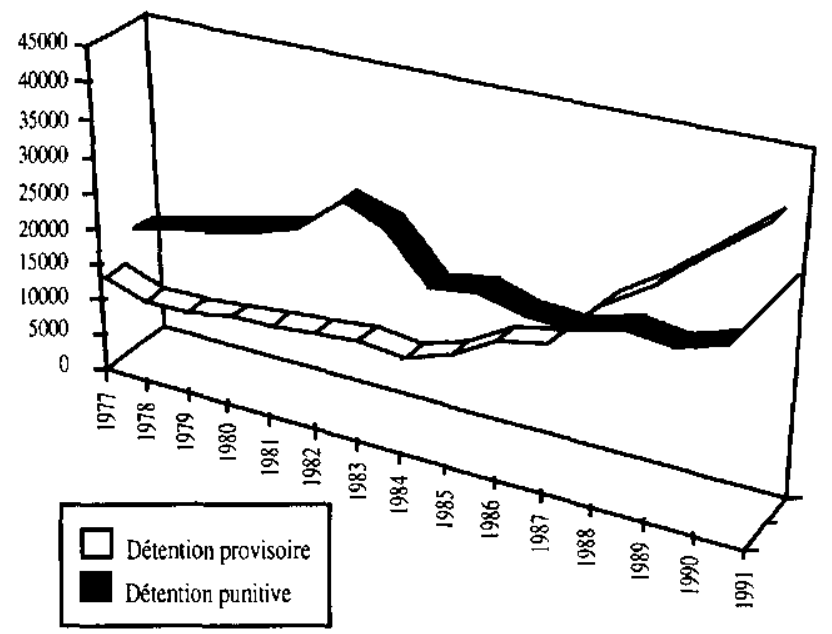

Source : Meloche (1993)

En ce qui concerne le nombre de personnes présentes dans les prisons un jour donné, les prévenus ne constituent toutefois que $25 \%$ en moyenne, soit le quart de la population carcérale au niveau provincial (figure 2). Cette disproportion entre les entrées des prévenus et leur présence au sein des prisons québécoises s'explique bien évidemment par la différence dans la longueur des séjours qui s'établit, en moyenne toujours, à 11,2 jours pour les personnes en détention provisoire (les prévenus) et à 34,0 jours pour celles en détention punitive (les condamnés ${ }^{9}$ ).

9. À l'exception des sentences fédérales, soit celles de 2 ans et plus, retranchées du calcul parce qu'elles sont purgées dans les pénitenciers. 


\section{Figure 2}

Distribution de la présence, à un moment donné, des prévenus et des détenus dans les établissements de détention du Québec, 1981-1991

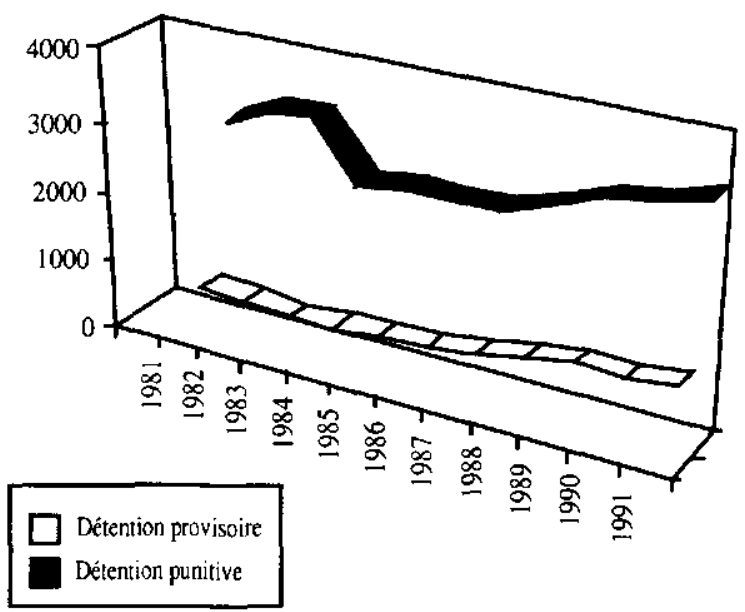

Source : Meloche (1993)

\section{UNE ANALYSE DES FLOTS}

La décision de mise sous garde revient dans un premier temps à la police, puis au tribunal. Détention policière et détention judiciaire constituent deux réalités suffisamment distinctes pour qu'il soit utile d'établir entre elles une différenciation sémantique tranchée. Je parlerai donc, dans le premier cas, de mise sous garde et, dans le deuxième cas, de détention provisoire (et non préventive ${ }^{10}$ ). Le choix du terme "provisoire" n'est

10. En effet, il nous semble utile de distinguer ici entre deux réalités souvent confondues sous le générique «détention préventive». Le Code criminel canadien définit ce qu'il faut entendre, au sens de la loi, par détention préventive : une mesure imposée au lieu de toute autre peine qui pourrait être infligée pour une infraction commise, sans précision quant à sa durée, le cas d'un justiciable placé en détention préventive faisant l'objet d'un examen par la Commission des libérations conditionnelles dès l'expiration d'un délai de 3 ans à compter du jour où le contrevenant a été mis sous garde. Cette étude est par la suite refaite tous les deux ans, puis tous les ans, en vue de déterminer l'éligibilité à la libération conditionnelle du justiciable considéré comme un délinquant dangereux. 
peut-être pas le plus adéquat ${ }^{11}$, il est néanmoins, à l'heure actuelle, le plus répandu. Nous nous plions à l'usage. Provisoire doit donc être pris ici dans le sens de «en attendant», autrement dit dans l'attente de l'issue des procédures.

Les données dont il sera question, dans les analyses qui suivent, concernent un échantillon représentatif de 1792 suspects appréhendés par les policiers de la SPCUM durant l'année 1989, et pour lesquels une demande d'intenter des procédures a été ultérieurement adressée à la Cour du Québec, chambre criminelle et pénale. Cet échantillonnage n'opérait aucune sélection en fonction de l'infraction ou de l'issue des procédures.

\section{La décision des policiers en matière de mise sous garde}

À la police, on découvre que plus de $90 \%$ des suspects pour lesquels on demande que des procédures soient adressées à la Cour du Québec, chambre criminelle et pénale, font l'objet d'une arrestation initiale qui les mène au poste de police. Pour certains, cette première forme de détention ne durera que le temps de procéder à certaines opérations d'usage (vérification d'identité, prise d'empreintes ou de photos, Alcootest...). Une fois ces formalités accomplies, l'agent ayant procédé à l'arrestation ou le fonctionnaire responsable ${ }^{12}$ évalue si l'individu peut ou non être remis en liberté moyennant une promesse ou un engagement à comparaître.

Parmi les personnes de l'échantillon ayant fait l'objet d'une arrestation, $29,1 \%$ seront ainsi remises en liberté en attendant leur première comparution au tribunal. Reste que la majorité des suspects $(70,9 \%)$ conduits au poste de police pour une arrestation suite à une infraction voient leur situation se transformer en une mise sous garde qui s'étend jusqu'au moment de leur première comparution à la cour, celle-ci devant normalement se tenir dans les 24 heures suivant la mise sous les verrous par les policiers. Si l'on ajoute à ceux-là les suspects au sujet desquels un mandat d'arrêt a été émis et qui sont éventuellement écroués entre le moment où la demande d'intenter des procédures est acheminée au tribunal et celui de leur comparution initiale devant le juge, on constate que près de trois quarts des inculpés $(72,5 \%)$ se présentent à cette première étape des procédures devant la Cour du Québec en tant que détenus.

11. Ph. Robert (1983:2) discute en effet, très pertinemment d'ailleurs, l'emploi de ce vocable pour désigner la détention avant et durant le procès. L'auteur attire l'attention sur le fait que : "provisoire, tout emprisonnement l'est au sens maintenant le plus courant de l'adjectif : quoique plus ou moins long, il doit cesser un jour au lieu de durer perpétuellement ».

12. Le Code criminel canadien définit le fonctionnaire responsable comme étant «le fonctionnaire qui, au moment considéré, commande le poste de police ou autre lieu où un prévenu est conduit après son arrestation». 


\section{La décision du juge à la comparution}

Un certain nombre des suspects comparaissant détenus devant le juge vont être remis en liberté illico, avec ou sans conditions. Mais, dans tous les cas, le juge peut choisir de procéder à une enquête sur cautionnement s'il estime qu'il serait ratsonnable de remettre le suspect en liberté mais qu'il considère, par ailleurs, qu'il lui faudrait des garanties supplémentaires que le prévenu ne tentera pas de se dérober à la justice.

La trajectoire suivie par le justiciable à partir du moment où il comparaît une première fois devant le juge peut donc prendre différentes directions. Certains embranchements se dessinent plus clairement que d'autres. Ainsi, les suspects comparaissant libres conserveront de facto, pour une très grande part (dans $91,5 \%$ des cas), leur liberté jusqu'à la fin des procédures, et seule une infime partie d'entre eux (soit $8,5 \%$ ) auront à subir une enquête sur cautionnement à l'issue de laquelle des proportions semblables se verront refuser (à $47,6 \%$ ) ou accorder (à $52,4 \%$ ) le droit de demeurer libres pour la suite des procédures, moyennant un engagement à respecter un certain nombre de conditions précisées par le juge ou, plus rarement, suite au dépôt d'une caution exigée par ce dernier.

En revanche, un plus grand nombre de suspects comparaissant détenus voient leur situation se modifier, la plupart $(56,0 \%$ d'entre eux) après la tenue d'une enquête sur cautionnement. Ainsi, parmi ceux qui ne subiront pas d'enquête sur cautionnement, $55,1 \%$ seront remis en liberté par le juge, alors que $44,9 \%$ verront la mise sous garde initialement imposée par les policiers se transformer en détention provisoire ordonnée par le juge. Pour ceux subissant une enquête sur cautionnement, les probabilités de remise en liberté $(50,1 \%)$ ou, au contraire, d'imposition d'une mesure de détention provisoire $(49,9 \%)$ s'équivalent.

Enfin, à tout moment de la procédure avant l'enquête sur cautionnement, le statut de l'inculpé est susceptible de changer. Cela est particulièrement vrai pour les suspects éventuellement remis en liberté. En effet, ce n'est qu'exceptionnellement qu'un suspect qui se voit imposer une mesure de détention provisoire à l'issue de l'enquête sur cautionnement est ultérieurement remis en liberté, les procédures le concernant n'étant pas terminées. Par contre, si à l'issue de la comparution ou de l'enquête sur cautionnement, $64,3 \%$ des suspects sont remis en liberté, ils ne sont plus que $56,1 \%$ à être libres au moment où est rendue la décision judiciaire sur leur cause. Trois situations sont alors susceptibles de s'être alors produites: 1) le suspect ne s'est pas présenté au tribunal Iorsque requis, de sorte qu'on a choisi de l'écrouer pour éviter qu'un tel désagrément ne se reproduise; 2) le suspect ne s'est pas conformé aux conditions rattachées à sa mise en 
liberté pour la durée des procédures ${ }^{13}$; 3) l'inculpé a commis une ou plusieurs nouvelles infractions.

Ce qu'il importe surtout de retenir, c'est qu'au moment où se prend la décision judiciaire sur la cause, $43,9 \%$ des inculpés se présentent détenus devant le juge, situation qui risque fort de leur être préjudiciable, comme nous le verrons. Reste à voir quels sont ceux, parmi les suspects, auxquels une telle mesure sera imposée.

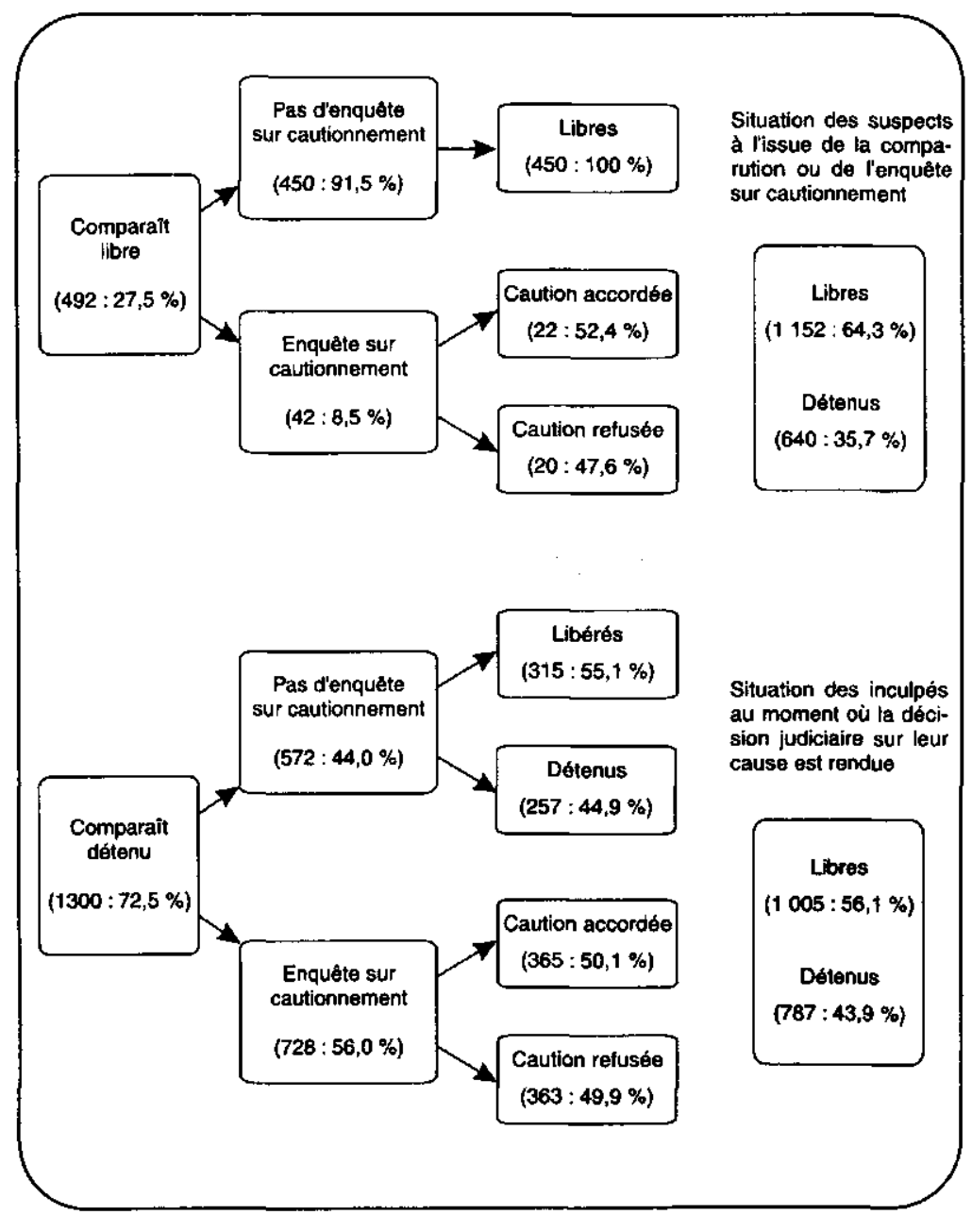

13. L'inculpé est alors non seulement susceptible de se retrouver sous les verrous pour cette raison, mais il est en outre passible de nouvelles accusations, nommément de bris de conditions. 


\section{CONCERNANT LES DÉTERMINANTS DE LA DÉTENTION PROVISOIRE}

\section{Des motifs d'incarcération très clairs...}

Au Canada, deux motifs répertoriés dans le Code criminel canadien ${ }^{14}$ permettent le recours à des mesures de détention avant et durant procès soit :

1) pour le motif principal que la détention est nécessaire pour assurer la présence d'un(e) suspect(e) devant le tribunal afin d'y être traité(e) selon la loi:

2) pour le motif secondaire que la détention est nécessaire dans l'intérêt public ou pour la protection de la sécurité du public, y compris toute probabilité marquée qu'une fois remis(e) en liberté, le ou la suspect(e) commette une nouvelle infraction criminelle ou cherche à nuire à l'administration de la justice.

Le premier motif est difficile à établir, à moins que le prévenu soit incapable de fournir une adresse, ou encore que l'on puisse prouver qu'il a déjà essayé de se soustraire à la justice. C'est donc dans une minorité de cas qu'il sera invoqué. Cela est toutefois moins vrai pour la police que pour le tribunal, puisque ce motif est utilisé par la police pour expliquer la mise sous garde de $49,2 \%$ des suspects, alors qu'il ne constitue plus que $21,3 \%$ des motifs d'incarcération appliqués par les juges pour justifier l'imposition d'une mesure de détention provisoire. La différence dans le recours au premier motif d'une instance à l'autre tient essentiellement au fait qu'entre le moment de leur arrestation et leur première comparution devant le tribunal, bon nombre de suspects, initialement incapables de fournir une adresse de référence, réussissent à pallier cet obstacle à leur remise en liberté en fournissant une adresse, qu'il s'agisse de la leur ou de celle d'un parent répondant ou même, éventuellement, de celle d'un quelconque refuge acceptant de se porter garant.

Le motif secondaire aux yeux de la loi, soit celui de la sécurité du public, devient donc le principal motif de mise sous garde en termes de proportion. $\grave{A}$ cette enseigne, une panoplie de situations s'associent à la notion de sécurité publique : nature de l'infraction, récidive confirmée ou escomptée de la part du suspect, problèmes psychiatriques ou de toxicomanie observés ou soupçonnés, voire attitude récalcitrante du suspect lors de l'arrestation. Landreville et Laberge (1992:36) indiquent clairement les difficultés que pose la décision de mise sous garde surtout lorsqu'il est question de sécurité publique : 
La décision est d'autant plus difficile qu'elle ne porte pas véritablement sur un comportement effectif (sauf dans les rares cas de crimes véritablement «crapuleux») mais sur la prédiction d'un comportement futur. Et si les notions qui reviennent le plus souvent sont celles d'intérêt public, de danger pour le public, de danger pour le justiciable lui-même... ces notions sont souvent présentées de façon fort vague, sans guide d'opérationnalisation susceptible de permettre aux décideurs de déterminer le sens précis et les comportements visés par la formulation particulière de la loi.

\section{Des prescriptions légales difficilement contournables}

S'ajoutent aux motifs généraux précités un certain nombre de prescriptions légales plus spécifiques qui obligent en quelque sorte la mise sous garde du suspect, à savoir :

1) un certain nombre d'infractions considérées comme particulièrement graves (essentiellement les infractions contre l'État et les crimes causant la mort d'individus);

2) les infractions relatives à l'importation, la fabrication et le trafic de drogues;

3) les situations où le justiciable contrevenant à la loi est déjà soumis soit à un ordre de garder la paix, soit à une sentence non encore échue : une ordonnance de probation, de détention (on comprend alors qu'il se trouve en liberté illégale) ou de libération conditionnelle ${ }^{15}$.

Néanmoins, ces obligations ne sont pas incontournables : le législateur a prévu ${ }^{16}$ que le juge de paix ordonne la mise en liberté du prévenu qui, répondant à ces critères, fait néanmoins valoir l'absence de fondement de sa détention sous garde. Cette disposition est toutefois rarement utilisée de sorte que, pour les justiciables répondant à l'une ou l'autre des caractéristiques précitées (et a fortiori ceux cumulant plusieurs d'entre elles), l'imposition d'une mesure de détention provisoire est prévisible et se justifie, à tout le moins au sens de la loi. Mais les détenus présentant de telles caractéristiques ne forment qu'un petit nombre (autour de 15\%) des personnes arrêtées et mises en accusation. Pour les autres, il faut chercher ailleurs le motif de leur détention.

\section{D'autres considérations}

Pour toute une série de situations autres que celles décrites plus haut, et lorsqu'il s'agit d'infraction objectivement moins graves (infractions sans victimes directes et, de manière générale, infractions contre les biens, 
celles-ci constituant $51,8 \%$ des causes entendues à la Cour du Québec et faisant partie de l'échantillon), ce sont essentiellement les caractéristiques personnelles et judiciaires du justiciable qui déterminent la mise en détention prédécisionnelle. L'espace qui m'est alloué ici ne me permet pas de faire la démonstration en long et en large de cette assertion. Je me verrai donc obligée de renvoyer le lecteur curieux à l'étude originale (Cousineau, 1992). Je me contenterai ici de tracer les grandes lignes des conclusions qui y sont rapportées (un tri de ces conclusions devant nécessairement avoir lieu). Dans un premier temps, il appert que pour un même type d'événement, l'utilisation de faux chèques ou le fait d'assener un coup de poing à un inconnu, par exemple, un individu qui présente de bonnes garanties de représentation (un emploi assuré, des revenus confortables, des relations familiales stables) et un casier judiciaire vierge - ou peu s'en faut - aura toutes les chances de comparaître libre devant le juge, alors qu'un autre plus jeune, sans emploi, sans conjoint risque fort de se retrouver détenu pour toute la durée des procédures, et ce, de manière encore plus certaine s'il s'avère qu'il possède des antécédents judiciaires, quels qu'ils soient, ou encore s'il est connu des policiers pour avoir des fréquentations douteuses (selon leurs propres termes).

Dans un deuxième temps, on se doit de constater que pour tous ceux accusés de contraventions à la loi somme toute triviales (troubler la paix, tapage, vagabondage ou même possession de petites quantités de drogues douces) et qui ne possèdent pas d'antécédents judiciaires (ce type de causes étant rarement entendues devant la Cour du Québec chambre criminelle et pénale, du moins dans le district judiciaire de Montréal - elles ne représentent que $5 \%$ des causes de l'échantillon), il devient, à toutes fins utiles impossible de déterminer les raisons faisant en sorte que les uns sont gardés sous les verrous alors que les autres peuvent continuer à jouir de leur liberté pour la durée des procédures, autrement qu'en se rattachant à des considérations anecdotiques.

Certains événements types donnent également lieu, plus couramment qu'à l'ordinaire, à la mise sous garde pour une partie ou pour la durée des procédures. Entre autres, les vols sous menaces se produisant dans des commerces, même s'ils rapportent à leurs auteurs des gains peu substantiels et n'occasionnent pas de blessures aux victimes, mènent directement en cellule à la police, mise sous garde qui se traduit dans $75 \%$ des cas par l'imposition d'une mesure de détention provisoire au tribunal. On peut croire qu'il s'agit là d'une situation dans une certaine mesure conjoncturelle : des pressions de la part du public pour une sévérité accrue des agents pénaux vis-à-vis de ce type d'infraction, en particulier, assureraient la mise sous garde des suspects quels qu'ils soient et quelles que soient les circonstances entourant l'affaire. 
Par ailleurs, deux groupes de contrevenants voient leur situation se modifier de manière importante à la comparution. Dans le premier cas, il s'agit, nous l'avons déjà mentionné, des suspects incapables d'indiquer où ils habitent au moment de leur arrestation. La plupart d'entre eux réussissent à fournir une adresse de référence avant de comparaître devant le juge, de sorte que leur détention initialement attribuée au premier motif (avéré par le manque d'adresse) n'a plus de raison d'être. Dans le deuxième cas, il s'agit des hommes soupçonnés de s'être livrés à des actes de violence conjugale. Deux considérations s'imposent ici : d'abord, il s'agit des causes les plus susceptibles d'avorter au tribunal faute de preuves; ensuite, les suspects impliqués dans ce type de situation présentent souvent - ironiquement - l'image reconnue en droit du bon père de famille : ils sont plus âgés que la moyenne des inculpés, ils occupent un emploi stable, jouissent de revenus réguliers, etc.

En somme, notre étude a montré - comme l'avaient fait avant, pour la France, celles d'Aubusson de Cavarlay (1987) et de Lévy (1987) - que la décision permettant au suspect de conserver sa liberté pour la durée des procédures repose en grande partie sur le fait que ce dernier est à même de fournir de bonnes garanties de représentation associé à une situation judiciaire peu compromettante. De ce fait, les suspects les plus démunis et les plus criminalisés - et de manière encore plus probante, les plus démunis fortement criminalisés - risquent davantage de se retrouver sous les verrous pour une partie ou pour la durée des procédures, et ce, quelle que soit l'infraction qui leur est reprochée dans l'immédiat.

\section{Des ambiguïtés du côté du mode d'emploi}

En matière de mise sous garde par la police, le législateur exprime clairement le devoir de l'agent de la paix - que les circonstances autorisent à procéder à une arrestation sans mandat - d'éviter dans toute la mesure du possible d'effectuer l'arrestation, et de délivrer à la place une citation à comparaître. Ce petit bout de phrase «dans toute la mesure du possible " le législateur le précise en notant qu'il s'agit pour l'agent 1) de voir s'il existe des motifs raisonnables et probables de croire que l'intérêt public ne peut être sauvegardé sans arrêter la personne; et 2) de s'assurer qu'il n'y a aucun motif raisonnable de croire que s'il n'arrête pas la personne celle-ci ne se présentera pas devant le tribunal pour y être traitée selon la loi. Autrement dit, l'agent de la paix peut se sentir fondé de procéder à l'arrestation s'il a la moindre raison de croire que le suspect ou bien peut représenter une menace pour la sécurité du public, ou bien risque de ne pas se présenter à son procès. Or, les chiffres le montrent sans équivoque, c'est bien plus souvent qu'autrement que l'agent estime que l'un ou l'autre de ces facteurs de risque est présent.

En matière de détention provisoire au tribunal, la Loi sur la réforme du cautionnement fixe les règles imposant au juge de paix l'obligation de 
mettre le prévenu en liberté, sauf dans le cas où le bien-fondé de la détention est démontré. Ainsi, le juge doit, à moins que le poursuivant ne le convainque de la nécessité du contraire, permettre au suspect de conserver sa liberté pour la durée des procédures. En outre, même si le poursuivant convainc le juge de paix de ne pas mettre le prévenu en liberté sur simple remise d'une promesse à comparaître qui ne serait assortie d'aucune condition, le Code criminel canadien comporte tout un arsenal de modalités flexibles pour la mise en liberté. En effet, les mécanismes suivants sont prévus : a) le prévenu remet une promesse assortie des conditions que le juge de paix fixe : $b$ ) le prévenu contracte, sans caution ni dêpôt d'argent, un engagement dont le montant et les conditions sont fixés par le juge de paix : c) le prévenu contracte, avec caution mais sans dépôt d'argent, un engagement dont le montant et les conditions sont fixés par le juge de paix.

Or, malgré toutes ces prévisions visant à favoriser la mise en liberté des suspects pour la durée des procédures judiciaires, il reste que la détention prévaut encore pour une partie fort appréciable d'entre eux (43,9\% des suspects traduits devant la Cour du Québec à Montréal, en 1989, se trouvaient détenus au moment où la décision judiciaire sur leur cause était rendue). On est alors fondé de s'inquiéter des conséquences de l'imposition d'une mesure de détention provisoire qui touche un si grand nombre d'inculpés.

\section{LE CARACTÈRE DÉTERMINANT DE LA MISE SOUS GARDE À LA POLICE...}

Traditionnellement, l'évaluation des impacts dans le champ de l'intervention pénale s'est restreinte à l'examen de l'issue des procédures. Mais notre étude a montré que l'enchaînement des maillons d'influence s'amorce bien avant. Un élément déterminant de la chaîne des événements consiste en effet dans la toute première décision prise par ie policier d'arrêter ou non le suspect pour le conduire au poste de police. À partir de là, on peut voir que près des trois quarts des suspects arrêtés et amenés au poste se retrouvent sous les verrous (mis sous garde) jusqu'au moment de leur première comparution devant le tribunal. Et, c'est. essentiellement dans ce bassin de suspects détenus au moment de leur première comparution devant le tribunal que se recrutent ceux qui demeureront détenus pour la durée des procédures. En effet, il est rare qu'un suspect libre au moment de sa première comparution au tribunal se voie imposer à cette étape une mesure de détention provisoire pour la suite des procédures, et ce, quelle que soit l'accusation qui pèse contre lui. Les inculpés au sujet desquels l'imposition d'une mesure de détention provisoire est jugée nécessaire sont donc, à plus de $95 \%$, des suspects détenus au moment de la comparution ou de l'enquête sur cautionnement, le cas échéant. 


\section{... ET LE CARACTÈRE DÉCISIF DE LA DÉTENTION PROVISOIRE}

Concernant le caractère décisif de la mesure de détention provisoire en tant que telle, celle imposée — ou plutôt, nous venons de le voir, le plus souvent maintenue - par le juge au tribunal, on a depuis longtemps identifié une relation significative entre le fait d'être gardé en détention et l'issue de la cause : les personnes détenues durant leur procès et qui, par conséquent, paraissent détenues (souvent menottes aux mains et parfois chaînes aux pieds) devant le juge au moment où la décision judiciaire sur leur cause est rendue sont plus souvent déclarées coupables et, lorsqu'elles le sont, sont non seulement plus souvent condamnées à des peines d'emprisonnement, mais à des peines d'emprisonnement de plus longue durée. Ces données se vérifient dans le cadre de notre étude.

Ainsi, on constate que parmi ceux qui continuent à jouir de leur liberté pour la durée des procédures, $54,4 \%$ plaident finalement coupables à l'une ou l'autre étape du processus judiciaire, alors que $7,6 \%$ sont reconnus coupables à l'issue d'un procès tenu habituellement devant juge seul, exceptionnellement devant juge et jury (moins de $1 \%$ des causes entendues devant la Cour du Québec chambre criminelle et pénale de Montréal au moment de notre étude). C'est donc $62,0 \%$ des inculpés libres qui sont, d'une façon ou d'une autre, reconnus coupables. Parmi ceux détenus pour la durée des procédures, $80,9 \%$ plaident coupables et $5,3 \%$ sont reconnus coupables, de l'une ou l'autre des manières citées plus haut. C'est donc ici $86,2 \%$ des suspects qui voient leur culpabilité reconnue.

Tableau 1

Décision judiciaire sur la cause selon le statut du suspect au moment aù la décision est rendue

\begin{tabular}{|c|c|c|c|}
\hline & \multicolumn{3}{|c|}{ Statut du suspect } \\
\hline & Libre & Détenu & Total \\
\hline \multicolumn{4}{|c|}{ Décision judiciaire } \\
\hline Plaide coupable & $\begin{array}{r}547 \\
54,4 \% \\
\end{array}$ & $\begin{array}{r}627 \\
80,9 \% \\
\end{array}$ & $\begin{array}{r}1174 \\
65,9 \% \\
\end{array}$ \\
\hline Reconnu coupable & $\begin{array}{r}76 \\
7.6 \% \\
\end{array}$ & $\begin{array}{r}41 \\
5,3 \% \\
\end{array}$ & $\begin{array}{r}117 \\
6.6 \% \\
\end{array}$ \\
\hline Acquitté & $\begin{array}{r}93 \\
9,3 \% \\
\end{array}$ & $\begin{array}{r}17 \\
2,2 \% \\
\end{array}$ & $\begin{array}{r}110 \\
6,2 \% \\
\end{array}$ \\
\hline Libéré & $\begin{array}{r}289 \\
28,8 \% \\
\end{array}$ & $\begin{array}{r}90 \\
11,6 \% \\
\end{array}$ & $\begin{array}{r}379 \\
21,3 \% \\
\end{array}$ \\
\hline Total & $\begin{array}{r}1005 \\
56,6 \%\end{array}$ & $\begin{array}{r}775 \\
43,4 \%\end{array}$ & $\begin{array}{r}1780 \\
100 \%\end{array}$ \\
\hline
\end{tabular}

Données manquantes : $12(0,7 \%)$

Source : Cousineau (1992) 
Concernant plus spécifiquement le plaidoyer de culpabilité, là où se retrouve la plus grande différence de proportions entre les accusés libres et ceux détenus pour la durée des procédures, il convient de souligner qu'une étude portant sur la négociation de plaidoyer, réalisée à partir d'entretiens menés après d'inculpés incarcérés en attendant l'issue de leur cause au tribunal (Gravel et Cousineau, 1989), a montré que les conditions de vie associées à la détention provisoire sont telles, dans bien des cas, qu'elles pousseraient nombre de suspects à plaider coupable rapidement (certains disent à «n'importe quel prix»), dans le seul but de faire cesser cette forme d'emprisonnement devenue intolérable.

Tableau 2

Sentence selon le statut du suspect au moment où elle est rendue

\begin{tabular}{|l|r|r|r|}
\cline { 2 - 4 } \multicolumn{1}{c|}{} & \multicolumn{3}{c|}{ Statut du suspect } \\
\cline { 2 - 4 } \multicolumn{1}{c|}{ Sentence } & \multicolumn{1}{c|}{ Libre } & Détenu & \multicolumn{1}{c|}{ Total } \\
\hline Probation & & & \\
\hline Prison & 421 & 93 & 514 \\
& $67,6 \%$ & $14,2 \%$ & $40,2 \%$ \\
\hline Prison + probation & 71 & 213 & 284 \\
\hline Amende & $11,4 \%$ & $32,5 \%$ & $22,2 \%$ \\
\hline Total & 37 & 334 & 371 \\
\hline
\end{tabular}

Données manquantes: $13(1,0 \%)$

Source : Cousineau (1992)

En matière de sentence, la relation qui se dessine avec le statut de l'inculpé est encore plus frappante. Ainsi, alors que $83,5 \%$ des suspects détenus jugés devant la Cour du Québec chambre criminelle et pénale, écopent d'une peine d'emprisonnement (seule dans $32,5 \%$ des cas, et assortie d'une période de probation dans $51,0 \%$ des cas), seulement $17,3 \%$ des suspects libres pour la durée des procédures subissent le même sort (sentence d'emprisonnement uniquement : $11,4 \%$ des cas et assortie d'une période de probation : $5,9 \%$ des cas).

D'aucuns pourraient arguer que ces données ne sont que le reflet du bon fonctionnement de l'appareil pénal : ne seraient ainsi retenus en détention provisoire que les vrais criminels, ceux contre lesquels il existe suffisamment de preuves pour présumer de manière assez certaine de l'issue de la cause : une reconnaissance de culpabilité, et contre qui il est raisonnable 
de vouloir se protéger pour la durée du procès, étant entendu qu'ils encourent de toute façon une sentence d'incarcération. L'argument se défend, il s'applique même certainement dans nombre de cas. Mais il ne suffit pas. Il ne permet pas d'expliquer le recours à la détention provisoire dans le cas de suspects somme toute peu criminalisés, accusés de vols simples $(48,8 \%$ maintenus en détention provisoire pour la durée des procédures), de vandalisme $(39,8 \%$ en détention provisoire), de recel $(37,5 \%$ en détention provisoire) et d'atteinte à l'ordre public ( $54,6 \%$ en détention provisoire), pour ne citer que quelques exemples. Or, ils feront aussi partie du lot de ceux qui seront reconnus coupables et se verront imposer une peine d'incarcération. Et que dire des suspects incarcérés pour la durée des procédures qui, à l'issue de celles-ci, ne reçoivent pas de sentence d'incarcération (ils comptent tout de même pour plus du quart $[27,7 \%]$ de ceux soumis à une mesure de détention provisoire). Pour tous ceux-là, à tout le moins, la question de la nécessité de la détention provisoire mérite d'être posée sans ambages puisque la nécessité de la détention punitive ne semble pas, elle, avérée.

\section{De certains usages détournés de la détention provisoire}

L'aspect certainement le plus problématique de la détention provisoire consiste dans le fait qu'elle s'applique à des personnes dont la culpabilité n'est pas établie et qui, jusqu'à preuve du contraire, devraient être présumées innocentes. $\grave{A}$ ce sujet, je reprends cette constatation de Ph. Robert (1986) déjà citée en avant-propos mais encore fort pertinente ici : «La détention avant jugement n'est pas une peine, seulement une mesure provisoire de sûreté ; malheureusement, il est bien difficile de la distinguer résolument en pratique de la peine d'emprisonnement ". D'autant plus qu'il semblerait, certains procureurs de la poursuite l'ont affirmé, qu'on utilise parfois la détention provisoire à seule fin de s'assurer que le prévenu, d'une quelconque façon, passe un minimum de temps en prison, notamment dans le cas de situations d'agression sexuelle ou de violence conjugale au sujet desquels il est moins que certains que l'issue de la cause se traduise par une décision de culpabilité ou, dans le cas contraire, par une peine carcérale. Aussi, devant l'incertitude de la peine, on chercherait à prolonger les procédures, de façon à faire passer le plus de temps possible au suspect à l'intérieur des murs de la prison à titre préventif, avant même que sa culpabilité ne soit reconnue, au cas où il s'en tirerait à titre punitif.

De leur côté, les policiers se serviraient aussi de la détention provisoire comme d'une mesure de sûreté. De fait, il y a tout lieu de croire que l'obligation faite aux agents de la paix de s'assurer de la présence du suspect devant le tribunal les pousse à se montrer extrêmement prudents au moment de décider des personnes pouvant être remises en liberté. Ainsi la maxime « dans le doute, on s'abstient» deviendrait plutôt dans ce cas « dans le doute (et plutôt que de se faire prendre en défaut), on incarcère ». 
Dans un tout autre registre, il appert que certains individus, parmi les sans-abri entre autres, rechercheraient l'incarcération uniquement afin de profiter d'un certain nombre d'avantages dont ils sont privés à l'extérieur : chaleur et nourriture régulière, soins de santé spécialisés, désintoxication... Ceux-là ne souhaitent pas être remis en liberté en attendant leur procès. On peut toutefois facilement concevoir qu'il faut trouver d'autres moyens de les aider.

Il s'agit là d'autant d'usages détournés de la détention provisoire sur lesquels on ne s'est jamais véritablement questionné, et encore moins prononcé, mais qui certainement mériteraient d'être étudiés de plus près.

\section{CONCLUSION}

La première constatation qui se dégage de cet exposé met en évidence le caractère non résiduel du recours à ces formes d'incarcération avant et durant le procès. Ainsi, malgré les critiques virulentes des auteurs qui, d'une façon générale, remettent en cause le bien-fondé de l'application de telles mesures privant le suspect de sa liberté, et malgré les énoncés de principe prônant la modération dans l'imposition de celles-ci, on a pu constater qu'on y recourt encore dans un grand nombre de cas.

En outre, on constate que les suspects faisant l'objet de mesures de détention prédécisionnelles sont, d'une part, ceux incapables de présenter des garanties de représentation suffisantes et, d'autre part, ceux qui, malgré des garanties de représentations suffisantes, ont commis des actes qui, de l'avis des agents pénaux, appellent nécessairement la mise en détention jusqu'à ce que l'issue de la cause soit connue. Ces constatations soulèvent la question du caractère discriminatoire de la mesure, du moins pour les premiers.

Enfin, il me paraîtrait malvenu de ne pas clore mon propos en insistant sur le fait que la détention provisoire constitue de fait une mesure dispendieuse, non seulement en termes de coûts monétaires (ce qui est néanmoins le cas, il faut bien le dire) mais aussi - et peut-être bien plus _ en termes de coûts sociaux (on se rappellera la discussion autour des coûts sociaux de l'emprisonnement menée par Landreville, Blankevoort et Pires [1981]), et tout cela pour des personnes dont on n'a pas encore établi la culpabilité. Aussi convient-il maintenant, me semble-t-il, de se questionner sur les façons de gérer différemment le doute quant à la présence d'une personne mise en accusation à son procès, ou encore quant à la peur, avant même que le fait soit établi, qu'un individu ayant constitué un danger pour la société puisse récidiver. 


\section{RÉFÉRENCES}

AUBUSSON de CAVARLAY, B. (1987), Les filières pénales: étude quantitative des cheminements judiciaires. Collection Déviance et contrôle social, $n^{\circ} 43$, Paris : CESDIP.

CARDINAL, C. (1989), Police et comparution devant le tribunal: différenciation sociale, légale et sexuelle. Les cahiers du GRAPPP, $n^{\circ} 8$. Montréal : Université de Montréal / Université du Québec à Montréal.

CASAVANT, L. (à paraître). L'emprisonnement à défaut de paiement d'amende: un élément dans l'approvisionnement des centres de détention du Québec. Montréal : Université de Montréal, École de criminologie.

COMITÉ CANADIEN DE LA RÉFORME PÉNALE ET CORRECTIONNELLE (1969), Justice pénale et correction : un lien à forger. (Rapport Ouimet). Ottawa : Imprimeur de la Reine.

COMMISSION DE RÉFORME DU DROIT DU CANADA (1988), Les mesures assurant la comparution, la mise en liberté provisoire et la détention avant le procès. Document de travail $n^{\circ} 57$. Ottawa : Commission de réforme du droit du Canada.

COUSINEAU , M.-M. (1992), Processus décisionnel et détermination des trajectoires judiciaires: analyse du cheminement d'une cohorte de justiciables. Montréal: Université du Québec à Montréal, département de sociologie (thèse de doctorat inédite).

COUSINEAU , M.-M., LABERGE, D., THÉORÊT, B. (1986), Prisons et prisonniers : une analyse de la détention provisoire durant la dernière décennie. Les cahiers du GRAPPP, $\mathrm{n}^{\circ}$ l. Montréal : Université de Montréal / Université du Québec à Montréal.

COUSINEAU , M.-M., LANDREVILle, P., LABERGE, D., THÉORÊT, B. (1995), Mise sous garde et remise en liberté provisoire à la cour du Québec, chambre criminelle et pénale à Montréal. Montréal : Centre international de criminologie comparée.

FRIEDLAND, M. (1965), Detention without rial. Toronto: University of Toronto Press.

GARCEAU, M.-L. (1988), La détention provisoire: une mesure discriminatoire. Les cahiers du GRAPPP, $n^{*} 5$. Montréal : Université de Montréal / Université du Québec à Montréal.

GOTTFREDSON, S. D., GOTTFREDSON, D. M. (1986), Prediction : an Overview of Selected Methodological Issues. in Gottfredson, D. M., Tonry, M. (eds). Prediction and classification. Crime dans Justice a Review of Research. Chicago: The University of Chicago Press. (Chapter 3, 21-51).

GRAVEL, S., COUSINEAU, M.-M. (1989), La pratique de la négociation de plaidoyer au palais de justice de Montréal. Montréal : Centre international de crirninologie de Montréal.

HATTEM, T. (1980), Prisons du Québec : prisons des pauvres. Montréal : Université de Montréal, École de criminologie (mémoire de maîtrise inédit).

LANDREVILLE, P. (1994), « Compensatory Work Program, a Way of Limiting Prison Use? The Québec experience. » The Howard Journal of Criminal Justice. $33: 3$, 236-245. 
LANDREVILle, P. (1969), Prédiction de la gravité de l'agir délinquant. Thèse de doctorat inédite. Montréal : École de criminologie, Université de Montréal.

LANDREVILLE, P. LABERGE, D. (1992), Détention sous garde et dangerosité. Les cahiers du GRAPPP, $n^{\circ} 12$. Montréal : Université de Montréal / Université du Québec à Montréal.

LANDREVILLE, P., BLANKEVOORT, V., PIRES, A. P. (1981), Les coâts sociaux du système pénal. Les cahiers de l'École de criminologie, Montréal: Université de Montréal.

LÉVY, R. (1987), Du suspect au coupable : le travail de police judiciaire. Genève : Éditions Médecine et Hygiène.

MELOCHE, K. (1993), L'évolution et les transformations des populations carcérales sous juridiction provinciale au Québec, de 1977 à 1991. Montréal : Université de Montréal (mémoire de maîtrise inédit).

MORDEN, C. P. (1980), A multivariate analysis of the factors associated with bait decisions by police. Masters thesis. Center of Criminology. Toronto : University of Toronto.

MORRIS, N., MILLER, M. (1987), Prediction of dangerousness in the criminal law. Research in brief. Washington, D.C. : U.S. Department of justice, National institute of justice.

NÉRON, J. (1988), La gestion pénale des cas de non-paiement d'amende : ''usage détourné de lemprisonnement. Les cahiers du GRAPPP, cahier $\mathrm{n}^{\circ} 3$. Montréal : Université du Québec à Montréal et Centre intemational de Criminologie comparée.

NUFFIELD, J. (1982), La libération conditionnelle au Canada: recherches en vue d'une normalisation des décisions. Ottawa: Solliciteur général du Canada, Division de la recherche.

ROBERT, P. (1982), « Le procès criminel : éléments d'une approche socio-juridique de la procédure pénale. »Criminologie, $15:$ I, p. 21-37.

ROBERT, Ph. (1986), "Un "mal nécessaire"? La détention provisoire en France.» Déviance et société, $10: 1$, p. 57-63. 